\title{
Depression, Disease and Disability: Application to Patients with Traumatic Spinal Cord Injury
}

\author{
F. K. Judd, MD, DPM, FRANZCP, D. J. Brown, FRACP, FACRM, \\ G. D. Burrows, MD, ChB, DPM, FRANZCP, FRCPsych \\ University of Melbourne, Department of Psychiatry, Spinal Injuries Unit, Austin \\ Hospital, Heidelberg, Victoria 3084, Australia.
}

\section{Summary}

Depressive symptoms are common in medically ill patients. Methodological difficulties of many of the studies demonstrating this do not allow differentiation of depressive symptoms from depressive illness. This paper examines representative samples of studies in the medically ill to highlight these problems. Data from a study of patients with traumatic spinal cord injury are used to demonstrate (1) ways of overcoming some of these difficulties and (2) the means of differentiating depressive symptoms and depressive illness. Difficulties of detection and diagnosis of depression in the medically ill are discussed.

Key words: Depression; Medical illness; Traumatic spinal cord injury.

The term depression may include a wide variety of mood states from normal to severely pathological with ill defined criteria to distinguish normal from abnormal. Depressive symptoms in the medically ill maybe normal, in the sense that both content and intensity are appropriate to the patient's situation, but at other times maybe pathological in nature. Sadness and grief maybe normal responses to painful life events associated with threatened or actual loss, and are to be expected in association with life-threatening, severely disfiguring and disabling illness, but must be distinguished from specific clinical syndromes of depression, which may require independent treatment. Studies of depression in the medically ill, have generally been flawed by major methodological problems including lack of clarity regarding definition of a case and the classification of depressive syndromes, the absence of assessment measures which have been standardised in the medically ill and the heterogeneity of subject populations studied. 


\section{Depressive symptoms, disease and disability}

Studies of depression in medical inpatients have consistently suggested that approximately one third report moderate depressive symptoms. Studies by Schwab et al. (1967), Moffic and Paykel (1975), Cavanaugh (1983), and Rodin and Voshart (1987) are examples of the many studies which have been reported in the literature and can usefully be examined to highlight some of the findings to date and the methodological problems.

Schwab et al. (1967) studied 153 inpatients with a variety of medical diagnoses (including psychiatric disorder) within 3 days of admission, and found $22 \%$ were depressed according to their score on the Beck Depression Inventory (BDI). Depression was associated with all types of medical illness and occurred in patients with all degrees of severity of illness.

Moffic and Paykel (1975) using the BDI as a screening instrument, to assess the prevalence of depression in 150 medical inpatients, found 36 of the 150 (24\%) patients scored $>14$ at the first interview (in the week after admission). Patients were then re-interviewed each week subsequently and a further 7 patients scored above the cut off at one of these subsequent interviews, giving a total prevalence for the whole inpatient admission of $28.7 \%$. For 9 patients, the depression preceded the symptoms of diagnosable physical illness, and for 6 patients the physical symptoms of illness appeared to be the direct consequence of depression, so that for only 28 of the 150 patients $(18.6 \%)$ did depression appear to be the consequence of the physical illness.

Cavanaugh (1983) administered the mini mental status examination (MMSE), the General Health Questionnaire - 30 (GHQ) and the BDI to 335 medical inpatients. Patients were described as 'a substantial number were quite ill or recovering from severe illness'. Interviews were conducted at different points in their hospital course. Thirty-two per cent had BDI $>14$. Two thirds of these had a score in the mildly depressed range (13-20).

Rodin and Voshart (1987) evaluated depressive symptoms in 115 patients with end stage renal disease (ESRD) awaiting cadaveric transplantation. Patients had been receiving dialysis for between 3 weeks and 11 years. Sixty three were receiving home dialysis and 52 subjects were undergoing haemodialysis or intermittent peritoneal dialysis in hospital. Assessment was by semi-structured interview and questionnaires including the BDI. The BDI was completed by 113 patients of whom $29(25 \cdot 7 \%)$ scored $>17$ and $40(35 \cdot 4 \%)>14$.

These 4 studies which all demonstrate that depressive symptoms are common in the medically ill, illustrate many of the methodological difficulties found in this area. All of these studies failed to differentiate depressive symptoms from clinically diagnosed depressive illness. All included heterogeneous patient samples and two (Cavanaugh 1983; Rodin and Voshart, 1987) did not standardise the time of assessment which, as demonstrated by Moffic and Paykel's (1975) findings may influence the prevalence of depressive symptoms.

\section{Depressive illness, disease and disability}

Studies assessing the prevalence of depressive symptoms, such as those described above, have not generally distinguished normal responses from specific clinical 
syndromes. It is important to identify and appropriately treat those who do suffer from a depressive illness (DSM III major depressive episode). Depressive illness is associated with specific morbidity e.g. poor self care, poor participation in or compliance with medical treatment, specific mortality (depression is the most common cause of suicide) and without treatment generally persists for many months. (Beck, 1969; Robins and Guze, 1972).

Differentiation of normal from pathological depression was examined by Bukberg et al. (1984) in a study of 62 cancer patients. They found that depressive symptoms as revealed by BDI score were common (33\% scored $>14 \mathrm{BDI}$ ), consistent with previous studies in other groups of medically ill patients. Psychiatric interview of patients revealed that only $24 \%$ of patients met the DSM III criteria for moderately severe major depressive episode (MDE) (mean BDI 19.6, mean Hamilton Rating Scale for Depression (HRSD) score 22.5).

We have attempted to further clarify the nature of depressive symptoms in the medically ill by studying the prevalence, phenomenology and response to treatment of depression in patients with traumatic spinal cord injury (Judd et al., 1989).

Seventy one patients with acute traumatic spinal cord injury were studied from the time of admission (within 24 hours of injury), throughout their acute medical and inpatient rehabilitation treatment. Initial assessment occurred at a uniform time in the course of their illness (10-14 days after admission) and included a semistructured psychiatric interview and completion of the HRSD and BDI. Patients were then seen weekly and reinterviewed if the BDI score was $>14$. This procedure allowed systematic evalution of the variation in depressive symptoms over time and delineation of depressive illness (DSM III MDE), from depressive symptoms as assessed by the BDI.

The 71 patients participating in the study ranged in age from 18-69 (mean 31.4) years. Fifty two were male and 19 female. Forty one were paraplegic and 30 quadriplegic.

Fourteen patients $(19 \cdot 7 \%)$ had BDI $>14$ at the time of initial interview, and a further $13(18 \cdot 3 \%)$ had BDI $>14$ at some future time during inpatient treatment. Only 1 of the patients with initial elevated score, continued to describe severe depressive symptoms and was subsequently diagnosed as suffering from a depressive illness. For the remaining 13 patients the dysphoric mood did not persist. Similarly the 13 patients with isolated elevated BDI scores did not persistently report dysphoric mood. In both these groups of patients it was considered that dysphoric mood was most likely to have been a response to the losses sustained as a result of the injury. Specific additional stresses identified in, but not confined to, this group of patients included separation from family (family members unable to visit due to distance of hospital unit from patient's home), poorly controlled pain and other medical complications.

The majority of patients $(\mathrm{N}=44,62 \%)$ described only mild depression, consistently scored $<14$ on BDI, and at clinical interview showed no evidence of depressive illness.

Fourteen patients persistently ( $>3$ weeks) scored $>14$ on BDI. Reassessment of this group of patients by the Liaison Psychiatrist revealed that all 14 were suffering from a major depressive episode. Diagnoses were made using modified DSM III criteria, as recommended by Endicott (1984). These criteria require 
sustained lowering of mood and associated features of depression (specified minimum number of symptoms present every day for at least 2 weeks). MDE occurred with similar frequency in paraplegics and quadriplegics (17 vs $23 \%$ ) and the male:female ratio of 5:2 was equal to the overall ratio of male:female patients treated for traumatic SCI. Personality, interpersonal and illness variables suggesting vulnerability to depression were more frequently identified in those developing MDE than in the other groups and have been described in detail elsewhere (Judd et al., 1989). Of note, only 1 of the 14 patients had a past history of MDE, 2 of dysthymic disorder, 1 of alcohol and 1 of polydrug abuse. For 2 patients concurrent crises (wife killed in accident, severe family conflict) may have increased the liability to develop a MDE.

While our study (Judd et al., 1989) has overcome some of the methodological problems which have plagued previous studies, it has failed to address others. For example, while all patients were suffering from traumatic SCI, the degree of disability due to the injury was variable. Secondly, no attempt was made in this study to determine how a given disability affected each individual, taking into account their defence mechanisms, coping strategies, vocational and leisure pursuits and interpersonal functioning. While these questions which pertain particularly to aetiological aspects of the depression remain to be answered, the study has clarified the relationship between depressive symptoms and depressive illness in this group of medically ill patients.

\section{Detection and diagnosis of depression}

The BDI has frequently been used as a screening instrument for depression in the medically ill. Cavanaugh et al. (1983) have suggested that it is a particularly useful instrument for such patients. These investigators subdivided the BDI to two subscales, affective/cognitive (items $\mathrm{A}-\mathrm{N}$ ) and somatic (items $\mathrm{O}-\mathrm{U}$ ), and examined responses in medically ill inpatients, patients with major depression and normal controls. They suggested that 6 of the 14 cognitive/affective symptoms-irritability, dissatisfaction, crying, discouragement with the future, difficulty with decisions and sadness, may be a common emotional response to illness and hospitalisation.

They found the 14 cognitive/affective symptoms were the best indicators for severity of depression with both number and intensity of the symptoms increasing as depression becomes more severe. Feeling like a failure, loss of interest in people, feeling punished and suicidal ideation, pronounced levels of dissatisfaction, difficulty with decisions and crying were good discriminators for severe depression. By contrast the frequency of somatic symptoms increased minimally as severity of depression increased but severity of somatic symptoms increased as depressive severity increased.

The findings of other investigators are consistent with those of Cavanaugh et al. (1983). Moffic and Paykel (1975) using the BDI found that while somatic preoccupations, anorexia and insomnia were present in both groups, they were more common in those who were depressed. Further, they found that most marked differences between the depressed and non-depressed groups were for sadness, pessimism, guilt, irritability and suicidal ideation. Similarly, using the HRSD, Bukberg et al. (1984) found the non-somatic symptoms of depressed mood, anxiety, suicidal ideation, sense of helplessness, hopelessness and worthlessness 
appeared more frequently in the depressed group. Conversely with the exception of insomnia, somatic symptoms occurred with equal frequency in both groups.

If it is accepted that the BDI is a useful screening instrument an appropriate cutoff score for a medically ill population must be established. Beck (1967) reported that a cut-off point of 14 differentiates effectively between depressives and nondepressives. While this criteria has been adopted in many studies of depression in the medically ill, it has been suggested by others that a cut-off score of 17 provides optimal sensitivity and specificity for the clinical diagnosis of major depression (Craven et al., 1986). Nielsen and Williams (1980) suggested that for the purpose of calculating prevalence in the medically ill a BDI score of 13 had an optimal combination of sensitivity $(0.79)$ and specificity $(0.77)$ while a cutting score of 17 (sensitivity 0.66 , specificity 0.84 ) was optimal for the identification of at least 'moderate' levels of depression.

Cavanaugh (1984) has suggested that the DSM II criteria for major depressive episodes provide a useful framework for the diagnosis of depression in medically ill patients, but stressed that emphasis should be placed on the cognitive/affective symptoms. In addition, reported symptoms must be carefully examined to ensure they are the result of depressed mood. For example, psychomotor agitation and retardation are reliable symptoms of depression but may also be caused by several organic conditions, notably delerium. Sleep and appetite disturbance are frequently present in hospitalised patients but maybe helpful in making a diagnosis of depression if severe and out of proportion to the illness and discomforts of hospitalisation.

Endicott (1984) has suggested two ways in which standardised diagnostic criteria maybe modified for use in medically ill patients. Firstly, the established criteria maybe used, as is, by simply not counting the items e.g. sleep, appetite, energy likely to be altered as a result of the medical illness. The result of this is to increase the severity of the criteria required to make a diagnosis of $\mathrm{MDE}$, increasing the potential for false negatives. Alternatively different symptoms maybe substituted for those most likely to be affected by the medical condition and its treatment. In this case a clear rationale for the modifications is required. In our study (Judd $e t$ $a l ., 1989$ ) we chose the first alternative suggested by Endicott (1984). Subsequently, we have developed a modified set of DSM III R criteria (Table) using Endicott's (1984) second alternative. In this modified set of criteria, we have placed greater

Table DSM III-R criteria for major depressive episode (MDE) modified for use with medically ill

At least five of the following, present for at least 2 weeks, representing a change from previous function.

1 Depressed mood, most of the day, nearly every day, as indicated by subjective account or observation by others

2 Frequent crying

3 Markedly diminished interest in self care, treatment and rehabilitation or family and friends

4 Feelings of worthlessness or failure, self reproach, inappropriate or excessive guilt, feeling of being punished

5 Suicidal ideation or attempt

6 Diminished ability to think or concentrate or severe indecisiveness

7 Anorexia or weight loss not explained by the illness, its treatment, or discomforts of hospitalisation

8 Insomnia not explained by the illness, its treatment or the discomforts of hospitalisation

9 Psychomotor agitation or retardation not explained or organic brain syndrome, the illness or its treatment 
emphasis on the cognitive/affective symptoms of depression as suggested by Cavanaugh et al. (1983). We have substituted 'frequent crying' for the DSM III R item 'fatigue or loss of energy' which is likely to be altered by medical illness. In addition we have modified the items relating to loss of interest, insomnia and weight loss to account for the influence of the medical illness.

\section{Conclusions}

Depressive symptoms frequently occur in medically ill patients and are readily detected by standard screening instruments such as the BDI. The detection of depressive symptoms in this way does not confirm that the patient suffers from a depressive illness. Rather the symptoms maybe a manifestation of a wide range of psychiatric disorders or represent a normal response to their illness.

The strength of the association between depression and medical illness, coupled with the known morbidity and mortality of depressive illness make it imperative to clarify the features of depression in these patients. Further studies in homogeneous patient samples, using standardised assessment measures designed to differentiate depressive symptoms from various depressive and other psychiatric syndromes in the medically ill are required. Such studies will serve to more accurately determine the prevalence of depressive illness in medically ill patients, and to clarify the role of disease and disability variables in the aetiology and maintenance of these disorders.

\section{References}

American Psychiatric Association 1987 Diagnostic and statistical manual of mental disorders. 3rd Edn. Revised. (DSM IIIR) Washington DC, APA.

AMERICAN Psychiatric Association 1980 Diagnostic and statistical manual of mental disorders. 3rd Edn. Revised. (DSM III) Washington DC, APA.

BECK AT, W ARD CH, MENDELSON M, MOCK J, ERBOUGH J 1967 An inventory for measuring depression. Archives of General Psychiatry 4:351-363.

BECK AT Depression Clinical, Experimental and Theoretical Aspects. New York, Harper \& Row Publishers Inc, 1969.

Bukberg J, Penman D, Holland JC 1984 Depression in hospitalised cancer patients. Psychosomatic Medicine 46:199-212.

Cavanaugh S 1983 The prevalence of emotional and cognitive dysfunction in a general medical population: Using the MMSE, GHQ and BDI. General Hospital Psychiatry 5:15-24.

Cavanaugh S, Clark DC, GibBons RD 1983 Diagnosing depression in the hospitalised medically ill. Psychosomatics 24:809-815.

Cavanaugh S 1984 Diagnosing Depression in the Hospitalised Patient with Chronic Medical Illness. Fournal of Clinical Psychiatry 45:13-17.

Craven J, Rodin G, Voshart K, Kennedy S 1986 Depression in the medically ill. Concordance of self report and clinician-rated measures with diagnosis of major depression. Psychosomatic Medicine 48:303-315.

ENDICOTT J 1984 Measurement of depression in patients with cancer. Cancer 53(Suppl)2243-2248.

JUDD FK, STONE J, WEBBER JE, BROWN DJ, BURROWs GD 1989 Depression following spinal cord injury: A Prospective Inpatient Study. British Fournal of Psychiatry 154:668-671.

MofFIC HS, PAYKel ES 1975 Depression in medical inpatients. British fournal of Psychiatry 126:346-353.

NiELSEN AC, Williams TA 1980 Depression in ambulatory medical patients. Archives of General Psychiatry 37:999-1004.

RoBINS E, GUZE S 1972 Classification of affective disorders. In: Recent advances in the psychobiology of the depressive illnesses. (Eds.) Williams TA, et al. Washington DC, Government Printing Office.

Rodin G, VoshaRT K 1987 Depressive symptoms and functional impairment in the medicall ill. General Hospital Psychiatry 9:251-258.

SCHWAB JJ, BIALOW MK, BROWN JMK, HolzER CE 1967 Annals of Internal Medicine 67:695-707. 